\title{
Du temps perdu au temps retrouvé : Proust face à
} Genette

Dairine O'Kelly

\section{OpenEdition}

12 Journals

Édition électronique

URL : https://journals.openedition.org/ml/245

DOI : $10.4000 / \mathrm{ml} .245$

ISSN : 2274-0511

Éditeur

Association Modèles linguistiques

Édition imprimée

Date de publication : 1 janvier 2012

Pagination : 69-98

Référence électronique

Dairine O'Kelly, « Du temps perdu au temps retrouvé : Proust face à Genette », Modèles linguistiques [En ligne], 65 | 2012, mis en ligne le 27 janvier 2013, consulté le 01 juillet 2021. URL : http://

journals.openedition.org/ml/245; DOI : https://doi.org/10.4000/ml.245 


\section{Du temps perdu au temps retrouvé : Proust face à Genette}

Dairine Ó Kelly

\section{Le cas Genette : Discours du récit. Essai de méthode (1972)}

\subsection{Expansion du verbe et récit, ou "Marcel devient écrivain "}

Discours $d u$ récit a longtemps servi de manuel de référence aux littéraires désireux de s'initier à ce qu'on appelait à l'époque la «nouvelle critique». Pour les littéraires, en effet, l'approche de Genette était rassurante, car tout en s'inscrivant dans la tradition de la "poétique » aristotélicienne, elle faisait appel aux nouvelles disciplines paralinguistiques alors en vogue, notamment à la sémiotique. Genette s'inspire également de la tradition anglo-saxonne représentée par Henry James et Percy Lubbock ${ }^{1}$. Discours du récit, qui prend comme seul domaine d'application l'œuvre de Proust, constitue une tentative pour bâtir ce que Genette appelle lui-même une « technologie du discours narratif ».

Mais il fait très peu appel à la linguistique à proprement parler. Dans sa bibliographie on trouvera donc Barthes, Greimas, Todorov, mais, à part Benveniste, aucun linguiste. L'essentiel de son analyse est cependant centré sur le temps ; autant dire qu'il n'a aucun fondement théorique, mais il réussit à donner le change grâce à un dispositif terminologique sophistiqué. Son appareil analytique est fondé sur les catégories empruntées à la grammaire du verbe par l'intermédiaire de Todorov $(1968)^{2}$. Afin de justifier cette utilisation des outils de la linguistique, il

1. Henry James, The Art of the Novel (recueil des préfaces à ses romans, 1907), Percy Lubbock, The Craft of Fiction (1921).

2. Les trois niveaux d'analyse sont présentés comme une reprise d'un travail antérieur de T. Todorov («Les catégories du récit littéraire », Communications 8 , 1968), qui établit une grille d'analyse centrée sur trois catégories :

(i) celle du temps, " où s'exprime le rapport entre le temps de l'histoire et celui du discours»; entrent dans cette catégorie ce que Todorov appelle les "déformations temporelles", c'est-à-dire le non-respect de l'ordre chronologique des événements et les relations d'enchaînement, d'alternance 
avance l'argument que, puisque «tout récit est une production linguistique assumant la relation d'un ou plusieurs événement(s)» (op. cit, 75), il peut être traité comme l'« expansion du verbe». Ainsi, des énoncés comme Je marche, ou Pierre est venu, seraient des formes minimales de récit; l'Odyssée n'est que l'amplification d'un énoncé comme «Ulysse rentre à Ithaque » et La Recherche du temps perdu l'amplification de « Marcel devient écrivain ». Et ainsi de suite ${ }^{3}$.

L'utilisation de l'analogie verbale amène Genette à distinguer «trois classes fondamentales de déterminations ", à savoir, respectivement :

(i) celles qui tiennent aux relations entre récit et diégèse, rangées sous la catégorie du temps,

(ii) celles qui tiennent aux modalités de la «représentation » narrative, donc aux modes de récit,

(iii) celles qui tiennent à « la façon dont se trouve impliquée dans le récit l'instance narrative, et, avec elle, ses deux protagonistes, le narrateur et son «destinataire réel ou fictif». À cette catégorie, il assigne le terme de voix, telle qu'elle est définie par Vendryès (aspect de l'action verbale dans ses rapports avec le sujet ; par « sujet », il faut entendre instance narrative).

ou «d'enchâssement » entre les différentes lignes d'action constitutives de l'histoire,

(ii) celle de l'aspect, « où la manière dont l'histoire est perçue par le narrateur », autrement dit le point de vue narratif,

(iii) celle du mode, c'est-à-dire «le type de discours utilisé par le narrateur » ; ceci recouvre l'opposition de la critique américaine jamesienne entre showing («représentation») et telling («narration »), les catégories platoniciennes de mimesis (imitation parfaite) et diegesis (récit pur), et les divers types de représentation du discours des personnages, les questions de présence et d'absence du lecteur et du narrateur. Genette organise ces catégories d'une manière un peu différente.

3. Genette semble ici être influencé par l'exercice scolaire du résumé. Sa réduction des romans à un énoncé minimal fait aussi penser aux présentations de films en une phrase, pour gens pressés, dans des magazines du type Pariscope. Partant de la conception du récit comme expansion du verbe dans un énoncé minimal, on peut imaginer un jeu de société pour public cultivé, qui consisterait à retrouver l'énoncé-source: "Résumez en une phrase les romans suivants: Madame Bovary, Anna Karénine, Jane Eyre, The Way of All Flesh, David Copperfield, Sister Carrie, Jude the Obscure, Le rouge et le noir, etc. ». Cela pourrait donner : Emma tousse plus haut qu'elle n'a la gorge ; Ah ! il fallait pas, il fallait pas qu'elle y aille ; Il ne faut jurer de rien ; Famille, je te hais ; David devient lui aussi écrivain ; Hurstwood devient clochard ; Jude devient à son tour écrivain ; Julien finit sur l'échafaud. 
L'essai de méthode de Genette est donc organisé autour de ces trois niveaux de définition du récit. Dans les trois premiers chapitres consacrés au «temps ", il étudie les problèmes qui relèvent de l'ordre, de la durée et de la fréquence. Un quatrième chapitre traite du mode, un cinquième de la voix.

\section{2. La catégorie du «temps »: (1) l'ordre}

Pour le traitement des problèmes regroupés sous la désignation "ordre », Genette est très influencé par la critique de cinéma, comme l'indique le passage suivant cité en guise d'introduction :

Le récit est une séquence deux fois temporelle [...] : il y a le temps de la chose racontée et le temps du récit (temps du signifié et temps du signifiant). Cette dualité n'est pas seulement ce qui rend possible toutes les distorsions temporelles qu'il est banal de relever dans les récits (trois ans de la vie du héros résumés en deux phrases d'un roman, ou en quelque montage " fréquentatif " de cinéma, etc. ; plus fondamentalement, elle nous invite à constater que l'une des fonctions du récit est de monnayer un temps dans un autre temps (Christian Metz, Essais sur la signification au cinéma, 1968, Klincksieck, Paris, 27).

Les différentes «discordances» entre l'ordre de l'« histoire» (ce par quoi j'entends la matière narrée) et celui du « récit » (selon moi, la mise en forme de cette matière par le narrateur) sont désignés par l'expression « anachronies narratives $»^{4}$, par rapport à « une sorte de degré zéro qui serait un état de parfaite coïncidence temporelle entre récit et histoire $»^{5}$.

Soit l'analyse du texte suivant, extrait de Jean Santeuil, que Genette propose comme exemple d'illustration (1972, 81 sq.) :

Quelquefois en passant devant l'hôtel il se rappelait les jours de pluie où il emmenait jusque-là sa bonne, en pèlerinage. Mais il se les rappelait sans la mélancolie qu'il pensait alors devoir goûter un jour dans le sentiment de ne plus l'aimer. Car cette mélancolie, ce qui la projetait ainsi d'avance sur son

4. En formant ce syntagme (la forme nominale correspondante étant anachronique) pour désigner tout décalage temporel, Genette a retenu la connotation associée à l'adjectif ("qui est déplacé de son époque ») ; le préfixe ana-emprunté au grec (« de bas en haut», puis «en arrière ", «à rebours", « de nouveau»), semble mal choisi pour entrer dans la composition d'un terme général qui doit aussi comprendre un mouvement prospectif. Dans le contexte de décalage temporel, le terme «rétrospection» peut servir pour désigner un regard rétrospectif attaché au sujet parlant, et le terme « ana-chronie » (avec trait d'union) pourrait servir pour désigner un mouvement semblable qui ne met en cause que l'objet, compte non tenu du regard.

5. La «manœuvre " narrative qui consiste à évoquer d'avance un événement ultérieur est appelé «prolepse »; le terme analepse est employé lorsqu'il s'agit d'« évocation après coup d'un événement antérieur au point de l'histoire où l'on se trouve". 
indifférence à venir, c'était son amour. Et cet amour n'était plus (Édition de la Pléiade, 674).

Dans ce passage, Genette repère neuf segments répartis sur deux positions temporelles, qu'il désigne par 2 (maintenant) et 1 (autrefois), et qu'il dispose de la manière suivante :

A 2 - «Quelquefois en passant devant l'hôtel il se rappelait » ...

B I — « les jours de pluie où il emmenait jusque-là sa bonne, en pèlerinage »...

C 2 - « Mais il se les rappelait sans »...

D 1 — «la mélancolie qu'il pensait alors »...

E 2 - «devoir goûter un jour dans le sentiment de ne plus l'aimer.» FI: «Car cette mélancolie, ce qui la projetait ainsi d'avance » ...

G 2 — « sur son indifférence à venir »...

H 1 — «c'était son amour » ...

I 2 - «Et cet amour n'était plus » ...

aboutissant à la formulation suivante :

$$
\mathrm{A} 2 \cdot \mapsto \mathrm{B} 1 \cdot \mapsto \mathrm{C} 2 \cdot \mapsto \mathrm{D} 1 \cdot \mapsto \mathrm{E} 2 \cdot \mapsto \mathrm{Fl} \cdot \mapsto \mathrm{G} 2 \mapsto \mathrm{H} 1 \mapsto \mathrm{I} 2
$$

Il procède ensuite à une définition des relations qui unissent les segments entre eux, autrement dit à une analyse syntaxique en termes de proposition principale et de proposition subordonnée. A est identifié comme le point de départ narratif en position autonome; B se définit par rapport à A comme "rétrospectif subjectif»; distinction est faite entre $\mathrm{l}^{\prime}$ «anticipation subjective » du présent dans le passé (E), et «anticipation objective» $(\mathrm{G})$, puisque Jean s'était trompé sur ses sentiments. Genette analyse les autres mouvements comme de simples retours à chacune des deux positions ( $\mathrm{H}$ et F). La distinction entre subjectif et objectif est laissée de côté, car elle relève de la catégorie qu'il examine dans la section traitant du «mode». La formule finale suivante fait apparaître les relations et les emboîtements, ainsi que l'incidence des rapports analeptiques (A) et proleptiques $(\mathrm{P})$.

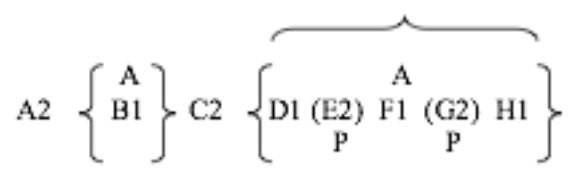

Il oppose «le temps de l'histoire» (erzählte Zeit — «temps du signifié » pour Metz) et le «temps du récit » (Erzählzeit — «temps du signifiant » pour Metz), qu'il considère comme un «faux-temps» ou «pseudotemps » (op. cit., 78). En fait, l'analyse de Genette ne distingue pas entre la pensée du personnage («se rappelait»), et ce à quoi renvoie la pensée. Elle ne prend en considération ni la valeur des sémantèmes (par exemple «se rappeler », ni celle des morphèmes (par exemple la désinence d'imparfait). 
Dans ce bref extrait de Jean Santeuil - l'exemple «idéal type » de Weinrich -, deux moments dans l'existence du protagoniste sont évoqués. Soit un premier moment identifié comme passé par rapport à l'instant d'écriture désigné par les noyaux prédicatifs suivants :

(i)a [...] il se rappelait (les jours de pluie) ;

(i)b [ ... ] il se les rappelait (les jours de pluie) ;

(i)c $\quad[\ldots]$ cet amour n'était plus.

Et un second moment, identifié comme antérieur au premier: et représenté par :

(ii)a [...] les jours de pluie où il emmenait sa bonne en pèlerinage ;

(ii)b $\underline{\text { Mais }}$ il se les rappelait sans la mélancolie qu'il pensait alors devoir goûter un jour dans le sentiment de ne plus l'aimer ;

(ii)c Car cette mélancolie, ce qui la projetait ainsi d'avance sur son indifférence à venir, c'était son amour.

$\mathrm{Du}$ point de vue de l'organisation du contenu informationnel, on constate que la deuxième et la troisième phrase sont reliées à la première par des connecteurs (mais et car) qui situent l'information, non par rapport à une successivité dans le temps, mais dans le cadre d'une argumentation. De ce point de vue, si la première phrase livre sous forme de complément d'objet ce dont le personnage se souvenait («des jours de pluie»), la seconde phrase apporte le complément de manière («sans mélancolie »), la troisième le complément de cause : (i) « [...] il se rappelait les jours de pluie [...] (ii) mais [...] sans mélancolie [...] (iii) car [...] cet amour n'était plus ».

Dans cet extrait de Jean Santeuil, le contenu du souvenir du personnage n'a aucun statut événementiel par rapport au récit, ce qui est confirmé par l'organisation syntaxique de l'information ${ }^{6}$. Cette façon d'organiser l'information est régie par le point de vue narratif qui semble être, en l'occurrence, celui d'un narrateur omniscient ayant accès à l'état d'âme de son personnage. Le sentiment de nostalgie qu'éprouve le personnage de Proust est, par sa nature même, associé au passé, mais il fait partie de son présent. Une véritable « analepse » implique une prise en charge par le narrateur, comme c'est le cas au début de La dame aux camélias d'Alexandre Dumas fils :

6. La nature paradoxale du souvenir, objet de discussion depuis les néoplatoniciens, est admirablement analysée par saint Augustin : «Mon enfance, par exemple, qui n'est plus, est dans un passé disparu lui aussi ; mais lorsque je l'évoque et la raconte, c'est dans le présent que je vois son image, car cette image est encore dans ma mémoire » (Livre XI. ch. XX). 
Il y avait en l'an de grâce 1845, dans ces années d'abondance et de paix où toutes les faveurs de l'esprit, du talent, de la beauté et de la fortune entouraient cette France d'un jour, une jeune et belle personne de la figure la plus charmante, qui attirait à elle, par sa seule présence, une certaine admiration mêlée de déférence pour quiconque, la voyant pour la première fois, ne savait ni le nom ni la profession de cette femme. Elle avait en effet, et de façon la plus naturelle, le regard ingénu, le geste décevant, la démarche hardie et décente tout ensemble d'une femme du grand monde. Son visage était sérieux, son sourire était imposant, et rien qu'à la voir marcher, on pouvait dire ce que disait un jour Elleviou d'une femme de la cour : «Évidemment, voici une fille ou une duchesse ».

Hélas! ce n'était pas une duchesse, elle était née au bas de l'échelle difficile, et il avait fallu qu'elle fût en effet belle et charmante pour avoir remonté d'un pied si léger les premiers échelons, dès l'âge de dix-huit ans qu'elle pouvait avoir en ce temps-là.

Cette ouverture offre un exemple intéressant pour ce qui est de la relation entre le temps de l'histoire et le temps du récit. On y trouve en effet, clairement explicités, tous les moments identifiés par Genette :

(i) le présent d'écriture: « Je me rappelle l'avoir rencontrée un jour ... »

(ii) le temps du récit : «Il y avait en l'an de grâce 1845 ... »

(iii) le temps de l'histoire, évoqué par analepse : «... elle était née au bas de l'échelle difficile .... »

(iv) coïncidence des trois temps (écriture, récit et histoire) : "Hélas! ce n'était pas une duchesse ... »,

On constatera la différence entre les passages de Jean Santeuil identifiés comme des "analepses", et, dans l'extrait ci-dessus de La dame aux camélias, le passage qui constitue une véritable parenthèse rétrospective prise en charge par le narrateur, comme le confirment son statut syntaxique d'indépendance et les temps et aspects des verbes.

Genette discerne, pour sa part, sous le terme récit, trois notions distinctes :

(i) «l'énoncé narratif, le discours oral ou écrit qui assume la relation d'un événement ou d'une série d'événements ». On retiendra les notions d'«énoncé », de " narration » et d'« événement ».

(ii) «la succession d'événements, réels ou fictifs, qui font l'objet de ce discours, et leurs diverses relations d'enchaînement, d'opposition, de répétition ». Cet emploi du mot tient compte uniquement du contenu, considéré comme une organisation d'événements successifs, abstraction faite de l'énonciateur, en d'autres termes, le récit en tant qu'« objet du discours ».

(iii) «l'acte de narrer en lui-même », il s'agit de l'acte d'énonciation considéré comme un événement. D'après ce sens du mot, la 
production du discours constitue en elle-même une action ( « raconter ses aventures est une action tout comme massacrer les prétendants de sa femme»)».

Genette déclare que son étude porte essentiellement sur le premier sens du mot, c'est-à-dire celui qu'il désigne sous le terme de discours narratif. Il assigne à ces trois sens les noms respectifs de récit, histoire et narration; l'analyse du discours narratif consiste ainsi en "l'étude des relations entre récit et histoire et récit et narration» (op. cit., 71-74). Il semblerait, malgré toutes ses précautions définitoires, qu'il se fourvoie. L'interprétation qu'il donne au terme « récit » implique une mise en forme particulière d'une série d'événements.

D'après cette définition, les problèmes de «paralepse » et d'«analepse» concernent la relation entre «récit» (ce que j'identifie comme la mise en forme) et «histoire » (la matière à narrer). L'« histoire», rappelons-le, n'existe que par le truchement du récit ; le temps qu'elle met en jeu implique un temps virtuel, porteur des événements, qu'on appellera temps d'univers. Celui-ci est calqué sur le temps d'expérience : on a l'expérience de la durée, de la fluence du temps selon une chronologie, une successivité : par exemple la succession des jours, des saisons, etc.). Il appartient au discours narratif (récit), qui retrouve ce temps d'expérience imaginaire par l'intermédiaire de l'histoire, d'agencer les événements selon un ordre qui réponde à ses besoins expressifs. Le récit est un agencement d'événements.

Le composant (l'unité) de base du récit est donc l'événement avec son temps propre (le temps d'événement) pris comme forme contenante, chargée de porter et de transporter une matière événementielle (contenue). On assiste là à un véritable jeu d'inclusions successives : temps d'univers contenant les événements ; événements contenus contenant à leur tour ce qui fait leur substance propre. On peut se demander dans quelle mesure Genette est conscient de ces distinctions. La suite du chapitre consacré à des problèmes d'ordre (83-90) concerne l'analyse d'un passage plus long (extrait de Sodome et Gomorrhe), conduit selon la même méthode. Cela permet à Genette d'introduire la notion d'« ellipse », définie comme "un bond en avant sans retour » ou « une simple accélération du récit ».

$\mathrm{Du}$ «micro-narratif », Genette passe ensuite à la «macro-structure » avec l'examen des 400 premières pages de La Recherche du Temps perdu, aboutissant à une formule qui, malgré l'ordre chronologique du macronarratif, fait apparaître, à l'intérieur des grandes articulations, « un vaste mouvement de va-et-vient à partir de la position-clé, stratégiquement dominante», des insomnies, identifiées comme "point d'émission mémorielle». Genette retrouve le modèle de ce «mouvement en zigzag » 
dans les six premières pages qui constituent ce qu'il appelle la «cellule embryonnaire ». Il considère que les sept débuts différents qu'il discerne dans les 200 premières pages, forment une série de prologues introductifs, et que cet exorde constitue une tentative d' "exorciser l'inévitable difficulté du commencement, laissant au narrateur le temps de placer sa voix ».

Deux nouveaux termes sont introduits. On parlera :

(i) de «la portée de l'anachronie », lorsqu'il s'agira de mesurer la distance temporelle qui sépare le terminus a quo (le moment où l'histoire est interrompue) et le terminus ad quem du mouvement, rétrospectif ou prospectif,

(ii) d'« amplitude » lorsqu'il s'agit de mesurer la durée contenue dans la prolepse ou l'analepse en question. À titre d'exemple, dans l'Odyssée, il cite l'analepse qui évoque les circonstances dans lesquelles Ulysse a reçu une blessure (chant XIX) comme ayant une "portée » de plusieurs dizaines d'années et une «amplitude» de quelques jours. Il pose comme principe que ce genre d'anachronie (flash-back en termes cinématographiques) constitue un récit temporellement "second", subordonné au récit dans lequel il s'insère (récit "premier»). L'étude détaillée de l'analepse et de la paralepse entraîne par la suite Genette dans ce qu'il appelle « les embarras ou les disgrâces de la terminologie » $(82$, n. 1), mais cela concerne essentiellement les structures narratives, non l'écriture stricto sensu.

\subsection{La catégorie du temps: (2) la durée}

La notion de temps $d u$ récit se heurte à quelques difficultés lorsqu'on confronte la "durée » du récit à celle de l'histoire racontée, parce que la durée du récit n'est autre que «le temps qu'il faut pour le lire». À juste titre, Genette conteste Ricardou, qui prétend qu'une scène dialoguée (à la supposer pure de toute intervention du narrateur et de toute ellipse) fournit «une espèce d'égalité entre le segment narratif et le segment fictif » : en effet, on ne peut pas restituer la vitesse à laquelle les paroles

7. En introduisant la notion de portée (sans doute la traduction du mot anglais scope, très à la mode à cette époque), Genette omet de parler de ce qui est, à mon avis, un paramètre essentiel, à savoir celui d'échelle: dans l'univers raconté de Jane Austen, un mois est un espace important de temps, 20 miles un parcours important spatial, ce qui n'est pas le cas, par exemple, ni chez Galsworthy (The Forsythe Saga), ni chez Roger Martin du Gard (Les Thibault).. Genette cite cependant (p. 132) Contre Sainte Beuve, où Proust fait l'éloge de la manière dont Flaubert opère des changements d'échelle temporelle dans l'Éducation sentimentale: "Ici un blanc et, sans l'ombre d'une transition, soudain la mesure du temps devient au lieu de quarts d'heure, des années, des décades $[. .$.$] extraordinaire changement de vitesse sans préparation ».$ 
ont été prononcées, ni les éventuels temps morts etc. ${ }^{8}$. Il propose donc un système déjà envisagé par Müller (1948) et Barthes (1967), fondé sur la notion de "constance de vitesse ", où vitesse renvoie au rapport entre une mesure temporelle et une mesure spatiale. Genette ne va pas jusqu'à prétendre que le récit "isochrone» («un récit à vitesse égale, sans accélérations ni ralentissements, où le rapport durée d'histoire/longueur de récit resterait toujours constant») puisse exister, mais on peut le poser comme un hypothétique degré zéro de référence. Et il fait observer que, si un récit peut se passer d'anachronies, « il ne peut pas aller sans anisochronies (effets de rythme) ».

À titre d'exemple, après avoir dressé la liste des grandes articulations narratives, il étudie les relations entre la durée de l'histoire et la durée du récit dans La Recherche pour en tirer deux conclusions :

i) sur «l'amplitude des variations, qui va de 190 pages pour trois heures à 3 lignes pour 12 ans »;

ii) sur son «évolution interne» - un ralentissement progressif du récit, caractérisé par l'importance croissante de scènes très longues couvrant une très petite durée d'histoire, et accompagné par la présence de plus en plus massive d'ellipses, en d'autres termes, une discontinuité croissante du récit.

Pour d'affiner son analyse, il rappelle à ce propos ce qu'il nomme les quatre mouvements narratifs («les formes canoniques du tempo romanesque ») à savoir :

(i) l'«ellipse », où un segment nul de récit correspond à une durée quelconque de l'histoire ;

(ii) la «pause descriptive», où un segment quelconque du discours correspond à une durée diégétique nulle ${ }^{9}$;

(iii) la «scène", le plus souvent dialoguée, qui réalise conventionnellement l'égalité du temps entre récit et histoire ;

(iv) le (récit) «sommaire » [traduit de l'anglais "summary" - pourquoi pas simplement « résumé » ?] qui, à la différence des autres, est un

8. Problèmes du nouveau roman, Seuil, Paris, 1967, 164. Ricardou utilise les termes narration et fiction, là où Genette parle de récit (narration) et histoire (diégèse).

9. Genette ajoute une note de bas de page, afin de préciser qu'il n'entend pas par là les « intrusions ou interventions de l'auteur », qui ne sont pas à proprement parler narratives. Il insiste sur le fait qu'il s'agit de pauses descriptives et non de descriptions qui, elles, peuvent être diégétiques (lorsqu'elles sont constitutives de l'univers spatio-temporel du récit) ou non. Cette distinction reste à élucider (je ne vois pas très bien ce qu'elle recouvre). 
mouvement variable couvrant « avec une grande souplesse de régime tout le champ compris entre la scène et l'ellipse ».

Ces quatre catégories renvoient à la tradition de la critique jamesienne reprise et développée dans le très beau livre de Percy Lubbock, The Craft of Fiction.

Tout en reconnaissant la nécessité d'identifier et de classer les diverses techniques (récit, commentaire, discours direct et indirect, etc.) que le narrateur a à sa disposition lorsqu'il s'agit de faire avancer son récit, je ne peux m'empêcher de me demander si le problème de la «durée » en général est correctement posé. L'approche de Genette, et celle de tous les critiques auxquels il renvoie, me donne l'impression qu'ils considèrent le «temps de l'histoire » comme une sorte de bande filmée en temps réel qu'il faudrait réduire à une durée de consommation pour le lecteur, un petit peu à la manière dont on réduit les romans et les essais dans le Reader's Digest ${ }^{10}$. C'est comme si l'histoire qui fait l'objet du récit avait réellement eu lieu dans le temps d'expérience. En fait, même si elle raconte une histoire vraie d'êtres qui ont réellement existé, dès qu'elle devient objet de discours, elle entre dans une autre dimension, elle est régie par d'autres lois, et son actualisation passe par le système de représentation et d'expression d'une langue donnée.

Il est intéressant de noter que les problèmes de la simultanéité et de la successivité, qui ont tant préoccupé un écrivain comme Virginia Woolf, ne sont même pas évoqués ${ }^{11}$. Aucun compte n'est tenu non plus de la

10. Voici par exemple ce qu'écrit P. Lubbock : «Not as a single form, but as a moving stream of impressions, paid out of the volume in a slender thread as we turn the pages - that is how the book reaches us; or in another image it is a procession that passes before us as we sit to watch. It is hard to think of this lapse and flow, this sequence of figures and scenes, which must be taken in a settled order, one after another, as existing in a condition of mobile form, like a pile of sculpture. Though we readily talk of the book as a material work of art, our words seem to be crossed by a sense that it is rather a process, a passage of experience, than a thing of size and shape." (1921 : 15).

11. En revanche, ces problèmes sont explicitement posés par J.-C. Chevalier dans une étude sur l'architecture temporelle d'un texte médiéval espagnol, le «Romancero tradicional». Il écrit notamment, à propos de la «matière temporelle» de six vers : «On se gardera de confondre ce qui est ici nommé «matière à exprimer » avec la réalisation des procès dans le monde physique. Ce serait faire du langage un simple appareil à photographier, à enregistrer. Ce serait le lier à l'ordre étroit et intangible de l'apparition des phénomènes, l'y réduire et, partant, condamner la pensée à la seule opération du constat. C'està-dire à n'être plus une pensée. Je peux fort bien en effet être devant deux procès que la réalité m'impose comme simultanéité : le fait de pleuvoir et celui 
différence entre la portée temporelle d'un état («être », «avoir», etc.) et celle d'un procès ( faire», «marcher», etc.), ni de l'image-temps qu'emportent les formes verbales exprimant les événements qui constituent la matière du récit. Du point de vue de la durée, comment traiter, par exemple, l'extrait déjà cité de La Dame aux Camélias ?

Il y avait en l'an de grâce 1845, dans ces années d'abondance et de paix où toutes les faveurs de l'esprit, du talent, de la beauté et de la fortune entouraient cette France d'un jour, une jeune et belle personne de la figure la plus charmante, qui attirait à elle, par sa seule présence, une certaine admiration mêlée de déférence pour quiconque, la voyant pour la première fois, ne savait ni le nom ni la profession de cette femme. Elle avait en effet, et de façon la plus naturelle, le regard ingénu, le geste décevant, la démarche hardie et décente tout ensemble, d'une femme du grand monde. [...]

Le premier chapitre, signé Jules Janin, est censé renvoyer à l'histoire qui donne lieu au récit. Cependant, l'existence de la jeune femme Mademoiselle Marie Duplessis - est traitée comme circonscrite temporellement dans l'année 1845. Quelle est, dès lors, la durée exprimée par le verbe avoir dans la phrase suivante : "Elle avait en effet, et de façon la plus naturelle, le regard ingénu, le geste décevant, la démarche hardie et décente tout ensemble, d'une femme du grand monde». Est-ce qu'on doit comprendre que ces qualités lui étaient attribuées uniquement pendant la durée de l'année 1845 ? La question est évidemment rhétorique. Notre pratique des conventions romanesques nous informe que la précision temporelle situe le moment où le narrateur découvre l'existence de la jeune fille, qui marque le moment où son histoire a commencé pour lui. Si je pose cette question, c'est uniquement dans le but

de se mouiller. Je ne suis pas obligé de m'intéresser à cette simultanéité. Il m'est permis de trouver plus de convenance à dire qu'il y a succession, et nécessaire ; que l'un est le résultat de l'autre. Deux prétérits en série y suffisent : «il plut, il se mouilla". La « matière à exprimer » peut être les simples ordres temporels du monde extérieur. Il n'est pas prescrit qu'elle ne soit que cela. Loin s'en faut » $(1971$, 55). Un autre problème vient se greffer ici, celui de la perception temporelle des procès dans le "monde physique » (ce que j'appelle le temps d'expérience) et, corrélativement, celui de la réalité temporelle, indépendamment de la perception qu'on en a. Deux questions pour résumer l'enjeu en le déplaçant légèrement: (a) y a-t-il un ordre en soi - un «ordre étroit et intangible", donc objectif — des procès dans le temps? (b) l'ordre perçu par le sujet pensant/parlant est-il un reflet de cet «ordre en soi », ou n'est-il pas déjà une vision subjective imposée par le filtre linguistique ? D'après les expériences réalisées par les psychologues, en particulier dans le cadre du constructivisme génétique de Piaget, il semblerait que le langage joue un rôle important dans la perception de la temporalité (pour une interprétation psychomécanique de ces expériences, cf. Joly 1990). On peut légitimement s'interroger sur la réalité $\mathrm{du}$ « temps de l'histoire ». 
d'attirer l'attention sur la série de faux problèmes posés par cette manière d'aborder l'analyse de la durée dans le récit.

\subsection{La catégorie du temps : (3) la fréquence}

Genette définit ce troisième paramètre comme «les relations de fréquence (ou simplement de répétition) entre récit et diégèse ». Il oppose le "singulatif» (on raconte «une fois ce qui s'est passé une fois»), et $l^{\prime}$ « itératif» (on raconte « en une seule fois ce qui s'est passé $n$ fois »). Dans le récit classique, observe-t-il, les segments itératifs sont presque toujours en état de subordination fonctionnelle par rapport aux scènes singulatives, auxquelles ils donnent une sorte de cadre ou d'arrière-plan informatif. Pour illustrer ce procédé, il cite le début d'Eugénie Grandet, qui commence par un long passage dit « itératif » dont le but est de préparer l'ouverture $\mathrm{du}$ récit à proprement parler. Ce long passage composé de plusieurs paragraphes offre une vue de Saumur qu' on peut dire "panchronique ", dans la mesure où sont évoquées différentes périodes de la vie de cette ville mélancolique, à présent («cette rue maintenant peu fréquentée ») et autrefois («des habitations trois fois séculaires » [...] l'histoire de France est là tout entière », etc.). Il s'agit en réalité, non d' "itérativité », mais d'une description qui transcende le temps et n'a rien à voir, ni avec le « singulatif» ni avec l'«itératif» tels qu'il les définit ci-dessus. Cette description sert de toile de fond - de fond de tableau - au récit " proprement dit », et qui est, lui, on ne peut plus « singulatif ${ }^{12}$ :

Il se trouve dans certaines provinces des maisons dont la vue inspire une mélancolie égale à celle que provoquent les cloîtres les plus sombres, les landes les plus ternes ou les ruines les plus tristes. Peut-être y a-t-il à la fois dans ces maisons et le silence du cloître et l'aridité des landes, et les ossements des ruines. La vie et le mouvement y sont si tranquilles qu'un étranger les croirait inhabitées, s'il ne rencontrait tout à coup le regard pâle et froid d'une personne immobile dont la figure à demi monastique dépasse l'appui de la croisée, au bruit d'un pas inconnu.

[...]

En 1819, vers le commencement de la soirée, au milieu du mois de novembre, la Grande Nanon alluma du feu pour la première fois [...]

Genette observe que le récit itératif, comme la description, est au service du récit "proprement dit», qui est le récit singulatif ${ }^{13}$. Il estime

12. Genette ne cite que la phrase qui commence «En $1819, \ldots$ ». Le paragraphe incipit que j'ai rajouté indique clairement que ce long passage introductif, même s'il enferme des fragments itératifs, est descriptif; le «technologue du discours narratif » ne semble pas savoir faire la différence entre un état et un procès.

13. Non argumentée, cette assertion est étonnante. Des points de vue linguistique et philosophique, elle n'est pas tenable. Si le récit, qui est une forme de langage, 
que Flaubert est le premier romancier à «émanciper le récit itératif de cette dépendance fonctionnelle », et il donne comme exemple d'illustration la vie d'Emma au couvent, ses jeudis à Rouen où, à son avis, « ce genre de récit prend une amplitude et une autonomie tout à fait inusitées ». Mais ce serait Proust qui en ferait vraiment un usage exceptionnel «par extension textuelle, par l'importance thématique, par le degré d'élaboration technique ». Les trois premières grandes sections de La Recherche du temps perdu sont essentiellement itératives, et ce n'est qu'à partir du premier séjour à Balbec que s'établit la prédominance du singulatif.

Cette analyse de Genette semble, sinon ignorer, du moins mettre entre parenthèses le fait que toutes nos capacités perceptives et conceptuelles sont fondées sur une opposition entre le général (l'«itératif », dit-il) et le particulier (le «singulatif»). Cette réalité psychique et cognitive est d'ailleurs reflétée dans l'architecture temporelle de la plupart des langues. Rappelons que la fonction principale du présent simple en anglais est de marquer un présent «itératif » (cf. Joly \& Ó Kelly, Grammaire systématique de l'anglais, 1990, 204-206).

Si l'on examine de près les nombreux exemples de ce que Genette identifie comme récit « itératif », on constate qu'une distinction importante lui échappe. D'un point de vue théorique, il convient de distinguer entre le général et le générique. Il y a une grande différence entre l'explicitation d'une répétition ("Quelquefois ... il se rappelait »), et un événement itératif représenté comme s'il n'avait eu lieu qu'une seule fois. Ce phénomène n'est pas du tout limité au discours narratif, il constitue l'expression normale d'une démarche de l'esprit consistant à abstraire d'une série d'expériences leur quintessence, ce qui permet, à la manière d'un accord modulant, de structurer la distance qui sépare ce que Genette appelle le «singulatif » et $l^{\prime} \ll$ itératif ».

\section{Proust et les intermittences du passé}

Soit l'extrait suivant de Proust, dans la version Grasset de 1913, cité et commenté par Genette (1972, 170-175) ; les imparfaits (aspect simple) y

était par essence «singulatif », cela voudrait dire que le langage n'est fait que pour dire le particulier et le singulier. Or précisément, l'économie du langage consiste à permettre l'expression du général et de l'universel. Dénommer, c'est pouvoir quitter l'horizon de l'individuel et se donner la faculté de saisir intellectivement le spécifique et le générique. La «querelle» de l'universel et du singulier traverse l'histoire de la pensée philosophique occidentale. Dans son Essay upon Human Understanding (1693), John Locke apporte la première solution rationaliste à ce problème. Cognitivement, l'expérience de l'itératif et du générique intervient très tôt dans le développement psychique. 
apparaissent en gras, les prétérits en italiques et soulignés (une seule forme, soulignée, est à la fois en gras et en italiques) :

Françoise avait trop froid pour rester immobile, nous allâmes jusqu'au pont de la Concorde voir la Seine prise, dont chacun, et même les enfants s'approchaient sans peur comme d'une immense baleine échouée, sans défense, et qu'on allait dépecer. Nous revenions aux Champs-Élysées ; je languissais de douleur entre les chevaux de bois immobiles et la pelouse blanche prise dans le réseau noir des allées dont on avait enlevé la neige et sur laquelle la statue avait à la main un jet de glace ajouté qui semblait l'explication de son geste. La vieille dame elle-même ayant plié ses Débats demanda l'heure à une bonne d'enfants qui passait et qu'elle remercia en lui disant: "Comme vous êtes aimable!» puis priant le cantonnier de dire à ses petits enfants de revenir, qu'elle avait froid, ajouta: "Vous serez mille fois bon. Vous savez que je suis confuse !». Tout à coup l'air se déchirait : entre le guignol et le cirque, à l'horizon embelli, sur le ciel entrouvert, je venais d'apercevoir, comme un signe fabuleux, le plumet bleu de Mademoiselle. Et déjà Gilberte courait à toute vitesse dans ma direction, étincelante et rouge sous un bonnet carré de fourrure, animée par le froid, le retard et le désir du jeu ; un peu avant d'arriver à moi, elle se laissa glisser sur la glace et, soit pour mieux garder son équilibre, soit parce qu'elle trouvait cela plus gracieux, ou par affectation du maintien d'une patineuse, c'est les bras grands ouverts qu'elle avançait en souriant, comme si elle avait voulu m'y recevoir. «Brava! Brava! Ça c'est très bien, je dirais comme vous que c'est chic, que c'est crâne, si je n'étais pas d'un autre temps, du temps de l'ancien régime, s'écria la vieille dame prenant la parole au nom des Champs-Élysées pour remercier Gilberte d'être venue sans se laisser intimider par le temps. Vous êtes comme moi, fidèle quand même à nos vieux Champs-Élysées; nous sommes deux intrépides. Si je vous disais que je les aime, même ainsi. Cette neige, vous allez rire de moi, ça me fait penser à de l'hermine ! » Et la vieille dame se mit à rire.

Cet extrait, ainsi que le long passage qui clôt $D u$ côté de chez Swann, où Marcel raconte ses promenades en hiver aux Champs-Élysées et au Bois de Boulogne ${ }^{14}$ a fait l'objet, au lendemain de la guerre, d'un commentaire de Robert Vigneron ${ }^{15}$ auquel Genette fait référence.

Vigneron, spécialiste de Proust, attribue à des « remaniements hâtifs » la «confusion inextricable» des temps (imparfait vs. prétérit) dans cette fin de Swann, et en particulier dans l'extrait cité ci-dessus. À l'occasion de la nouvelle édition de ce texte chez Gallimard en 1919, Proust aurait en

14. Ce long épisode est extrait de la troisième partie de $D u$ côté de chez Swann, intitulée "Noms de pays : le nom "; il correspond aux pages 482 à 511 de l'édition Grasset; ancienne édition de la Pléiade (celle qu'utilise Genette) : pp. 394-417 ; édition Tadié de la Pléiade (1987) : pp. 387-420. J'utilise cette dernière, ainsi que l'édition Gallimard de 1919 (tome II, pp. 237-281).

15. «Structures de Swann : prétentions et défaillances», Modern Philology, Nov. 1946. 
effet mis bout à bout des épisodes initialement séparés. D'où, selon Vigneron, certaines difficultés chronologiques :

Pour dissimuler cette confusion chronologique et psychologique, l'auteur s'efforce de camoufler des actions uniques en actions répétées et barbouille sournoisement ses verbes d'un badigeon d'imparfaits. Par malheur, non seulement la singularité de certaines actions en rend invraisemblable la répétition habituelle, mais encore par endroits de tenaces passés définis échappent au badigeon et révèlent l'artifice.

Voilà donc Proust devenu apprenti plâtrier. Sans doute un de ces «jeunes » dont Paul Souday, dans Le Temps, déplore qu'ils «ne sachent plus du tout le français $»^{16}$. Un quart de siècle après Vigneron, mais «dans un autre langage », Genette déplore lui aussi cette sournoise tentative de camouflage, heureusement vouée à l'échec par la courageuse résistance de quelques passés définis :

[...] il arrive parfois que la relation échappe à toute analyse, et même à toute définition, le récit passant d'un aspect à l'autre sans se soucier de leurs relations mutuelles, et même apparemment sans les percevoir. [...] Comme le note Vigneron dans un autre langage, le premier paragraphe [...] est itératif, ses verbes sont à l'imparfait de répétition : «Au paragraphe suivant, écrit Vigneron, («Françoise avait trop froid ... »), les imparfaits et les passés simples se succèdent sans raison apparente [...]». [...] Convenons [commente Genette] que dans cet "état", le texte répond assez bien à la description sévère qu'en donne Vigneron : les formes itératives et singulatives s'y enchevêtrent d'une manière qui laisse l'aspect verbal dans une totale indécision » (1972, 171-172).

Il est « indéniable », poursuit-il, que La Recherche est faite de matériaux « hétéroclites », que c'est par conséquent un "manteau d'Arlequin ». « Il y a du «collage», ou plutôt du «patchwork» dans La Recherche [...] » (op. cit., 174). Il y en a aussi, entre autres, chez Braque, Miro et Picasso. Qui songerait, sans preuves à l'appui, à leur refuser cohérence et unité de vision $^{17}$ ?

16. Dès la publication de ce premier volume, Paul Souday, après avoir relevé plusieurs cas d'incorrections (en réalité attribuables à des coquilles de l'imprimeur) écrit en effet dans Le Temps du 10 décembre 1913 : «Il nous semble que le gros volume de M. Marcel Proust n'est pas composé, et qu'il est aussi démesuré que chaotique [...] ». En 1946, Vigneron s'inscrit donc dans une tradition déjà bien établie, perpétuée, on va le voir, par Genette. Proust avait immédiatement protesté contre les attaques de Souday. Il écrit notamment dans une lettre: "Il serait $[\ldots]$ assez extraordinaire que j'ignorasse les règles de l'accord des temps. Je vous assure que si le «vieil universitaire» que vous proposez d'adjoindre aux maisons d'édition n'avait à corriger que mes fautes de français, il aurait beaucoup de loisirs » (voir Pléiade 1987 : 1052).

17. Genette n'en reste d'ailleurs pas là. Après avoir déploré l'absence d'une marque de «singulatif », comme une fois, pour introduire l'extrait ci-dessus, (p. 35), il 
Les arguments de critique externe (réduction de la troisième partie de Swann par rapport au manuscrit, révision du texte à l'occasion d'une nouvelle édition, redistribution éventuelle d'épisodes, etc.) ne prouvent rien, surtout si l'on songe que Proust avait déjà l'expérience de la critique et de la traduction, c'est-à-dire qu'il était parfaitement conscient des problèmes de composition et d'écriture. Pour en rester sur le plan de la critique externe, on pourrait tout aussi bien, et plus légitimement semblet-il, se demander si un écrivain qui, en 1920, écrit sur le style de Flaubert un article où il analyse si finement la valeur de l'imparfait, aurait été, un an avant, à l'occasion de la nouvelle édition de Swann, ignorant des possibilités de la langue au point de commettre de grossières erreurs sur l'emploi des temps dans son propre texte.

Passons à la critique interne. Dans l'extrait cité, Proust reprend un seul temps, «Tout à coup l'air se déchirait» devenant «Tout à coup l'air se déchira ». Rien d'autre n'a été repris, par exemple «Nous revenions aux Champs-Élysées » (1913) reste inchangé en 1919, alors qu'il aurait pu aisément être remplacé par revînmes. De même dans « ... c'est les bras grands ouverts qu'elle avançait en souriant, ... », l'imparfait ne cède pas la place au prétérit, alors que le contexte y invitait ( $\underline{\text { se laissa }} \underline{\text { s'écria) }}$. Est-ce un fait de hasard ? Genette et Vigneron ne se posent pas la question.

Mais ce dernier a sa petite idée. Il observe, par exemple, que les verbes du passage qui précède cet extrait désignent un «imparfait d'habitude », alors que les passés simples indiquent, sans transition, un événement unique. La conclusion qui s'imposerait est que tout se passe «comme si l'auteur, incapable d'adopter définitivement un point de vue plutôt que l'autre, avait laissé inachevées ses transpositions temporelles » (c'est moi qui souligne $)^{18}$.

Examinons les faits de plus près, c'est-à-dire dans l'immanence du texte. On constatera d'abord que Vigneron et Genette n'ont pas pris en

commente en ces termes le paragraphe qui suit immédiatement : « Le troisième paragraphe, lui, porte une telle indication: «Le premier de ces jours ...» (qualifiée par Vigneron de "pénible raccord", mais habituelle chez Proust: " ainsi, à l'auberge de Doncières », II, p. 98, où « le premier jour » adjoint une illustration singulative à une amorce de tableau itératif). Mais cette indication ne peut valoir rétroactivement pour le deuxième, dont elle ne fait qu'aggraver par contraste l'indétermination » (op. cit. : 175, n. 1).

18. Cité par Genette 1972, 172). Cette façon d'aborder les grands textes me fait penser à un professeur à la section d'anglais d'une université située à l'extrémité occidentale de la Bretagne qui jugeait les pièces de théâtre d'après le critère de l'« ironie dramatique ». Il n'en trouvait pas trace dans The Playboy of the Western World; par conséquent, le chef d'œuvre de Synge ne pouvait pas être une grande pièce. 
considération l'expression lexicale de la temporalité (syntagmes nominaux, verbes, adverbes, conjonctions). Or celle-ci joue un rôle très important dans ce texte, par sa densité et sa fonction. De plus, elle est indissociable de l'expression grammaticale par les temps verbaux.

Une troisième dimension échappe totalement aux deux interprètes de Proust: la sémantèse verbale. Voici donc, ci-dessous, la structure temporelle lexicale de l'ensemble de cet épisode (237-273 de l'édition Gallimard, 387-414 de la Pléiade 1987), où Proust aurait fait preuve de tant de négligence. Il s'agit, je le rappelle, de l'évocation de ses rencontres avec Gilberte au Bois de Boulogne et aux Champs-Élysées ${ }^{19}$ :

- Un jour, comme je m'ennuyais à notre place familière ... (237)

- Retournerait-elle seulement aux Champs-Élysées ? Le lendemain elle n'y était pas ; mais je l'y vis les jours suivants ... (239)

... une fois où elles ne se trouvèrent pas en nombre ... (239)

... et je jouai désormais avec elle chaque fois qu'elle était là. Mais ce n'était pas tous les jours ; il y en avait où elle était empêchée de venir ... (239)

... Ces jours-là, elle annonçait d'avance qu'on ne la verrait pas ... (239)

... Du moins ces jours-là, je savais que je ne la verrais pas, tandis que d'autres fois ..., et le lendemain elle disait ... (239)

... il y avait aussi les jours de mauvais temps où ... (239)

- Aussi si le ciel était douteux, [ = quand / chaque fois que ... ] (240)

... Si je voyais la dame d'en face ... (240)

... Tout d'un coup, ... je ne voyais pas ..., mais je sentais ... (240)

... Un instant après, le balcon était pâle ... (240)

- ... depuis le jour où elle était apparue sur notre balcon, comme l'ombre même de la présence de Gilberte qui ..., dès que j'y arriverais me dirait ... (241)

- Et jusque dans ces jours où ... quand celle-ci cessait de tomber, ... alors tout d'un coup ... le soleil apparu entrelaçait des fils d'or. (241)

... Ce jour-là nous ne trouvions personne ... (241)

... une dame d'un certain âge qui venait par tous les temps, toujours harnachée ... (242)

... Car Gilberte allait tous les jours la saluer ... (242)

... elle lisait toujours les Débats ... (242)

[S'insère ici le paragraphe cité intégralement ci-dessus : «Françoise avait trop froid ... »].

- Tout à coup l'air se déchirait / se déchira ... (242)

... Et déjà Gilberte courait... (242)

19. La pagination est celle de l'édition Gallimard de 1919. Les espaces entre les séries d'occurrences indiquent les changements de paragraphes ; un retrait indique que la phrase est au début du paragraphe. 
... un peu avant d'arriver à moi, elle se laissa glisser et, ... c'est les bras grands ouverts qu'elle avançait en souriant... (243)

- Le premier de ces jours - [...] - ce jour fit pourtant faire un progrès à mon amour ... (243)

... cela me semblait aussi touchant que si, un de ces jours où elle était invitée à une matinée, elle y avait renoncé pour venir me retrouver ... (244)

... et tandis qu'elle me mettait des boules de neige dans le cou, je souriais ... (244)

... Bientôt..., ses amies arrivèrent ...

... Ce jour que j'avais tant redouté fut au contraire un des seuls où ... (244)

- ... pourtant ces moments où j'étais auprès d'elle et que depuis la veille j'avais si impatiemment attendus ... n'étaient nullement des moments heureux ... car c'étaient les seuls moments de ma vie ... et elle ne découvrait pas en eux un atome de plaisir. (245)

- Tout le temps que j'étais loin de Gilberte ... (245)

... elle avait souvent prétendu ... (245)

... elle m'avait donné souvent des marques apparentes de froideur ... (245)

... Mais à l'époque où j'aimais Gilberte ... (246)

- Mais quand j'arrivais aux Champs-Élysées - [...] - dès que j'étais en présence de cette Gilberte Swann ... avec qui j'avais joué hier ..., aussitôt tout se passait comme si ... (247)

... Par exemple si depuis la veille je portais dans ma mémoire ..., la figure de Gilberte m'offrait maintenant ... (247)

... Tandis que je m'apprêtais à profiter de cet instant désiré ... que j'avais préparée avant de venir ... dans les longues heures où j'étais seul ..., le même moi qui m'avait fait la saluer avant que je l'eusse identifiée, ... jusqu'à l'heure où elle s'en allait ..., ou de garder le silence pendant lequel j'aurais pu enfin ..., les progrès décisifs sur lesquels j'étais chaque fois obligé de ne plus compter que pour l'après-midi suivante. (248)

... Un jour que nous étions allés ... (248)

- Une autre fois, ... je lui avais demandé ... (249)

... et le soir je lui avais adressé un petit télégramme ... ce nom de Gilberte que j'avais tant de fois tracé sur mes cahiers. (249)

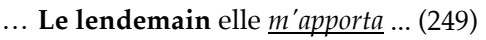

... Mais dans l'adresse de ce pneumatique - qui. hier encore n'était rien, ... et qui, depuis qu'un télégraphiste l'avait remis au concierge de Gilberte ..., un des petits bleus qu'elle avait reçus ce jour-là ... violettes ceintures symboliques de la vie, qui pour la première fois ... (249)

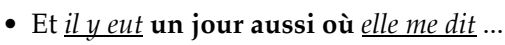

... Pourtant elle continua encore un moment à se contenter de me dire « vous". et comme je le lui faisais remarquer ... (249)

... Et me souvenant plus tard de ce que j'avais senti alors, ... tandis que son regard, ... m'atteignait... (250)

- Mais au moment même, je ne pouvais apprécier 
... Même après être rentré à la maison je ne les goûtais pas, car chaque jour, la nécessité qui me faisait espérer que le lendemain j'aurais la contemplation exacte ... de Gilberte, ... pour quelles raisons elle avait dû me le cacher jusqu'ici, ... le bonheur que je n'avais pas encore rencontré. (251)

- Si elle me donnait parfois de ces marques d'amitié, ... et cela arrivait souvent les jours mêmes sur lesquels j'avais le plus compté ... (251)

... quand - entrant dès le matin au salon ..., j'avais appris, ... que l'hiver recevait jusqu'au soir la visite inopinée et radieuse d'une journée de printemps. (251)

... Pendant que nous déjeunions, ... (251)

... Au collège, à la classe d'une heure ... une invitation à la fête où je ne pourrais arriver avant trois heures, jusqu'au moment où, ... (251)

... Hélas! aux Champs-Élysées je ne trouvais pas Gilberte, elle n'était pas encore arrivée. (252)

... je m'attendais à tout moment ... (252)

- ... persuadé qu'elle ne viendrait plus, quand, la fillette à la voix brève se jetait sur moi ... (252)

... Pendant que je montais l'avenue des Champs-Élysées, ... (252)

... elle m'était décochée en plein cœur, à quatre heures au lieu de deux heures et demie, ... (253)

... C'était aussi ce mystère qui me troublait quand, ... (253)

... M. Swann qui venait un peu après ... (253)

... une préoccupation si constante que les jours où, comme ceux-là, M. Swann (que j'avais vu si souvent autrefois ..., quand il était lié avec mes parents) ... (254)

... Ses relations..., quand j'en entendais parler à Combray ..., prenaient maintenant pour moi quelque chose de merveilleux, ... (254)

- ... il m'avait pourtant vu bien souvent à la campagne ... (254)

... depuis que j'avais revu Gilberte ... les idées sur lesquelles j'embranchais maintenant son nom étaient différentes des idées dans le réseau desquelles il était autrefois compris et que je n'utilisais plus jamais quand j'avais à penser à lui ... notre invité d'autrefois ... ce fut avec un sentiment de honte et le regret de ne pouvoir les effacer que je retrouvai les années où, aux yeux de ce même Swann qui était en ce moment devant moi ... et à qui ... je m'étais si souvent le soir rendu ridicule ... pendant qu'elle prenait le café avec lui ... (255)

... cette main que Philippe VII avait si souvent retenue dans la sienne, tandis que ... (255)

- Un de ces jours de soleil ..., je n'eus pas le courage ... (256)

- ... les mots où Gilberte avait laissé éclater sa joie de ne pas venir de longtemps aux Champs-Élysées. (256)

... Mais déjà le charme dont se remplissait mon esprit dès qu'il songeait à elle ... (256)

... Et quand vint l'heure du courrier, je me dis ce soir-là comme tous les autres : «Je vais recevoir une lettre de Gilberte, ... » ... (257)

- Tous les soirs je me plaisais à imaginer cette lettre, ... (257

... Tout d'un coup je m'arrêtais effrayé. (257) 
... Et dès lors, je m'efforçais ... (257)

- ... moi, qui avais d'abord aimé Gilberte, dès Combray, ... je pensais maintenant ... que de cette mienne vie ... , Gilberte pourrait devenir un jour l'humble servante, ... qui le soir, ... (258)

... à cause de qui j'avais d'abord aimé Gilberte, avant même de l'avoir vue, maintenant c'était surtout à cause de Gilberte que je l'aimais. (258)

... dans les moments où celui-ci ne m'apparaissait plus que comme un néant,... avant que Gilberte ne me connût ... (259)

... Et tandis que mon amour attendant sans cesse du lendemain l'aveu de celui de Gilberte, annulait, défaisait chaque soir le travail mal fait de la journée, ... (259)

... avec le même désespoir que les jours où je ne la voyais pas. (259)

... ce besoin unique de voir Gilberte qui faisait que des mois d'avance je ne pensais qu'à tâcher d'apprendre à quelle époque elle quitterait Paris ... , et ne désirant que rester toujours à Paris tant que je pourrais la voir aux ChampsÉlysées ... (260)

... Quand elle m'assurait parfois qu'elle m'aimait..., moins qu'elle ne m'aimait la veille ... (260)

... elles le persuadaient que le lendemain ne serait pas différent de ce qu'avaient été tous les autres jours ... (261)

... Alors dès le lendemain (ou attendant ... un de ces jours qui ne sont pas pareils aux autres, où le temps recommence ... (261)

- J'avais toujours à portée de ma main ... (261)

- ... je me le répétais mentalement sans cesse ... (262)

... Ce nom de Swann ..., que je connaissais depuis si longtemps, était maintenant pour moi ... (262

... Il était toujours présent à ma pensée ... (262)

... allait avoir enfin pour effet de faire brusquement entrer Gilberte venant à tout jamais vivre avec nous. (263)

... jusqu'au jour où je dis que ... (263)

- ... je passais tout mon temps à table ... (263)

... Et une fois, ma mère, en train de nous raconter, comme chaque soir à dîner, les courses qu'elle avait faites dans l'après-midi ... (264)

... Quelle mélancolique volupté. d'apprendre que cet après-midi-là ... (264)

... Mon père disait que je ... n'écoutais pas quand on parlait des conséquences politiques que ... (264)

... quand je tremblais d'amour devant lui aux Champs-Élysées, ... certaines particularités de notre vie d'autrefois, ... (265)

... au moment où il l'avait vue ... (265)

- Aussi après avoir trouvé la maison bien située. ils parlaient d'une autre qui l'était mieux,... et s'ils avaient eu un moment l'air d'être du même avis que moi, c'était par un malentendu qui ne tardait pas à se dissiper. (266)

- Les jours où Gilberte m'avait annoncé qu'elle ne devait pas venir ... (266) Parfois j'amenais Françoise ... (266) 
... si sur le chemin j'apercevais leur vieux maître d'hôtel ..., j'attachais sur ses favoris blancs des regards ... (266)

- Puis. nous poursuivions notre route ... (267)

... D'autres fois nous allions sur les boulevards ... (267)

- Mais le plus souvent - quand je ne devais pas voir Gilberte $-\ldots$ je dirigeais Françoise du côté du Bois de Boulogne. (267)

... puis. quand je me rapprochais ... (268)

- ... quand j'apercevais Mme Swann à pied ... (269)

... après que j'avais forcé Françoise ... (269)

... au fond de laquelle reposait avec abandon Mme Swann, ses cheveux maintenant blonds ... (270)

... Quand passaient des inconnus ... (270)

... Et les jours où je me sentais le courage de passer à côté d'elle ... (271)

... À un moment en effet. c'est dans l'allée des piétons, ... que j'apercevais Mme Swann ... (271)

... Parfois pourtant. quand elle s'était retournée ... (271)

- ... interrogeaient quelquefois un passant ...

- Mon cœur battait d'impatience quand je pensais qu'il allait se passer un instant encore avant que tous ces gens ... vissent le jeune homme inconnu ... saluer ... cette femme ... (272)

... Mais déjà j'étais tout près de Mme Swann ... (272)

... Certains jours ..., il m'arrivait de la rencontrer dans l'allée de la Reine Marguerite ... (273)

Reprenant Vigneron avec d'autres termes, Genette rend donc compte de ce passage en appliquant sa grille interprétative où il oppose le « singulatif » à l'«itératif ». L'itératif, ou fréquentatif, rappelons-le, s'applique à des formes qui désignent des «actions répétées » ou la «fréquence de l'action ». Quelques-unes des questions qu'on peut se poser à propos de cet épisode de la Recherche sont les suivantes :

$1^{\circ}$ ) L'expression lexicale et l'expression grammaticale du temps emportent-t-elles ici l'impression de « répétition » ou de «fréquence »?

$2^{\circ}$ ) Étant donné son sens d'intention déclaré, Proust veut-il exprimer l'itératif, par contraste avec le singulatif, ou bien recherche-t-il autre chose?

$\left.3^{\circ}\right)$ Y parvient-il et comment ?

À première vue, il semble bien qu'il y ait effectivement une opposition entre :

(a) l'expression du «singulatif »: évocation d'événements uniques marquée, lexicalement, par des locutions situant ceux-ci dans le temps et, grammaticalement, par des prétérits dont la fonction est d'en donner une vue perfective ( Un jour ... quand ... une fillette ... lui cria ... ; le premier de 
ces jours ... ce jour fit .... ; ce jour ... un des seuls où je ne fus pas trop malheureux; un de ces jours de soleil, je n'eus pas le courage ... ; un jour aussi où elle me dit ... ; jusqu'au jour où je dis que ... ; une fois où elles ne se trouvèrent pas en nombre ... ; une autre fois ... le lendemain elle $m^{\prime}$ apporta ... ; bientôt ... ses amies arrivèrent ... ; tout à coup l'air se déchira »)

(b) l'expression de l'«itératif»: évocation d'événements multiples, «répétés» ou «fréquents», du moins si l'on s'en tient aux seules apparences. La forme verbale utilisée est alors l'imparfait, en combinaison avec divers syntagmes adverbiaux classés traditionnellement comme "fréquentatifs" : "Il y avait aussi les jours de mauvais temps où son institutrice ... ne voulait pas ... ; ... qui venait par tous les temps, toujours harnachée ... ; car Gilberte allait tous les jours la saluer ... ; ... elle lisait toujours Les Débats ... ; ... elle avait souvent prétendu ... ; ... j'étais chaque fois obligé de ... ; car chaque jour ... cette même nécessité me forçait à ... ». Et de même avec parfois, souvent, chaque soir, sans cesse, tout le temps.

Mais s'agit-il véritablement d'une opposition entre le singulatif et l'itératif ? Une série de faits amènent à en douter. On observera d'abord que, dans ce long épisode, il ne se dégage aucune chronologie précise. Prenons un exemple précis - qui donne en fait la tonalité de l'ensemble-, celui d'une occurrence de « ce jour-là » qui, hors contexte, semble devoir désigner un jour précis repéré dans le temps, donc un «singulatif ». En fait, le contexte dans lequel il apparaît ne laisse aucun doute sur sa valeur :

Et jusque dans ces jours où toute autre végétation a disparu, où le beau cuir vert qui enveloppe le tronc des vieux arbres est caché sous la neige, quand celle-ci cessait de tomber, mais que le temps restait trop couvert pour espérer que Gilberte sortît, alors tout à coup, faisant dire à ma mère : «Tiens voilà justement qu'il fait beau, vous pourriez peut-être essayer tout de même d'aller aux Champs-Élysées», sur le manteau de neige qui couvrait le balcon, le soleil apparu entrelaçait des fils d'or et brodait des reflets noirs. Ce jour-là nous ne trouvions personne, ou une seule fillette prête à partir qui m'assurait que Gilberte ne viendrait pas (241; Pléiade, 390).

On remarquera qu'on n'est ici en présence ni d'un singulatif ni d'un itératif. Il s'agit d'une sorte de généralisation exemplaire. Genette parle à propos de ce type d'emploi de «totale indécision» $(1972,172)$ et d'« indétermination » $(1972,175)$. Il faut en convenir, mais à condition de ne pas le faire négativement; car, selon lui, telle indication temporelle (par exemple, «Le premier de ces jours ... », 243, Pléiade, 391), « ne fait qu'aggraver» ladite indétermination. Pourquoi l'indétermination seraitelle « grave »? Et grave pour qui ? Par rapport à quels canons serait-elle une erreur? Et si elle correspondait effectivement à la visée d'effet de Proust? On est du même coup conduit à se demander quel rôle joue l'imparfait par contraste avec le prétérit. Comment expliquer certaines 
combinaisons entre les indications lexicales de la temporalité et son expression temporelle? Autant de questions que Genette ne se pose pas, se contentant toujours de déclarer qu'il s'agit soit de "singulatif», soit d'« itératif ».

Prenons le cas de tout à coup avec son entourage co-textuel. On a vu qu'en 1919 (242 ; Pléiade, 391), Proust remplaçait l'imparfait par un prétérit (Tout à coup l'air se déchirait / se déchira). Mais dans d'autres cotextes, le même syntagme adverbial fonctionne avec l'imparfait :

Tout d'un coup, ... je ne voyais pas ..., mais je sentais ... (240 ; Pléiade, 389) ... alors tout d'un coup ... le soleil apparu entrelaçait des fils d'or ... (241; Pléiade, 390)

Tout d'un coup je $m^{\prime}$ arrêtais effrayé. (257 ; Pléiade : 402)

Le changement de la p. 242 (édition Gallimard) est dû à la fois à la sémantèse verbale («se déchirer » est un verbe lexicalement perfectif) et au co-texte élargi (série d'événements relatés au prétérit : «nous allâmes ... demanda l'heure ... remercia ... ajouta ... . L'air se déchira ... elle se laissa glisser ... se mit à rire»").

Mais l'imparfait choisi en 1913 n'a rien d'aberrant; il s'intègre parfaitement dans une autre série d'événements évoqués à l'imparfait: « avait trop froid ... les enfants s'approchaient... nous revenions aux Champs-Élysées ... je languissais de douleur ... l'air se déchirait ... je venais d'apercevoir ... et déjà Gilberte courait ... parce qu'elle se trouvait plus gracieuse ... c'est les bras grands ouverts qu'elle s'avançait ... ». Cette seconde série offre une autre vision des événements. C'est en fait cette seconde vision qui domine tout au long de l'épisode des Champs-Élysées et qui n'a rien à voir avec l'itération.

Comme je l'ai en effet indiqué plus haut, l'opposition n'est pas ici entre le singulatif et l'itératif, mais entre le spécifique et le générique, celui-ci englobant celui-là, tous deux entrant dans une subtile relation dialectique. Proust ne me paraît pas être préoccupé par le contraste de l'unique et du multiple (tel événement singulier vs. une série d'événements répétés), mais par la sélection, en quelque sorte emblématique, d'un événement particulièrement mémorable sur un fond d'événements identiques. Ceci est particulièrement sensible dans les deux exemples suivants :

Un de ces jours de soleil qui n'avait pas réalisé mes espérances, je n'eus pas le courage de cacher ma déception à Gilberte. (256 ; Pléiade, 401)

... (ou attendant une fête s'il y en avait une prochaine, un anniversaire, le nouvel an peut-être, un de ces jours qui ne sont pas pareils aux autres, ... ) (261; Pléiade, 405) 
Dans l'un et l'autre cas, il s'agit bien en effet de retenir, soit en l'actualisant, soit en le laissant dans le virtuel, un jour choisi dans une même série (les jours de soleil, les jours qui ne sont pas pareils aux autres) ${ }^{20}$. Cependant, l'identité de «ce jour-là » (ou de " ce soir-là », v. n. 19) n'est établie par rapport à aucun repère. Les autres épisodes narrés ne sont pas davantage repérés dans le temps : "un jour, une fois, une autre fois ... ». Ils se situent n'importe où dans cet espace temporel non structuré qui correspond à un moment - laissé lui-même dans l'indistinction — de la vie d'enfant du narrateur ${ }^{21}$.

Cette indétermination apparaît encore dans les faux repères que constituent des expressions comme «le lendemain», «la veille», «le soir », « les jours suivants », «l'après-midi suivante », ou des indications temporelles marquant un début: "désormais ", « dès lors », "depuis le jour où »; en réalité ni les unes ni les autres ne renvoient à un instant de référence spécifique et précis : dans tous les cas, en effet, le référent temporel auquel il est renvoyé (p. ex. «ce jour-là ») est lui-même indéterminé. Il s'agit donc là, en quelque sorte, d'une indétermination au second degré (soit par exemple: «les jours suivants (indétermination 1) de ce jour-là (indétermination 2)».

À tout cela s'ajoute l'effet cumulatif du temps rêvé (v. ci-dessus la note 20). Car, dans cette évocation des promenades enfantines, l'imaginaire assumé comme tel - joue un rôle capital. Proust a fréquemment recours à la modalité hypothétique, d'où l'accumulation des «si » (en anglais un if équivalent à "whenever") et des « conditionnels » dont l'effet est d'abstraire de l'actuel, de généraliser au maximum et d'accroître ainsi l'indétermination temporelle. En voici quelques exemples :

20. Et de même: «Et quand vint l'heure du courrier, je me dis ce soir-là comme tous les autres ... » (257 ; Pléiade: 401$)$.

21 Ce long épisode des Champs-Élysées et du Bois de Boulogne est introduit par le passage suivant, qui le précède immédiatement : «On devait se contenter de m'envoyer chaque jour aux Champs-Élysées, sous la surveillance d'une personne qui m'empêcherait de me fatiguer et qui fut Françoise, entrée à notre service après la mort de ma tante Léonie. Aller aux Champs-Élysées me fut insupportable. Si seulement Bergotte les eût décrits dans un de ses livres, sans doute j'aurais désiré de les connaître, comme toutes les choses dont on avait commencé par mettre le «double » dans mon imagination. Elle les réchauffait, les faisait vivre, leur donnait une personnalité, et je voulais les retrouver dans la réalité ; mais dans ce jardin public rien ne se rattachait à mes rêves » (237; Pléiade : 386 ; c'est moi qui souligne). L'épisode tout entier est donc placé sous le signe de l'indétermination que Genette reproche tant à Proust - une indétermination accentuée par l'imagination et le rêve. J'y reviens ci-dessous. 
Aussi si le ciel était douteux, dès le matin je ne cessais de l'interroger et je tenais compte de tous les présages. Si je voyais la dame d'en face qui, près de la fenêtre, mettait son chapeau, je me disais ... ( 240 ; Pléiade, 388).

Tous les soirs je me plaisais à imaginer cette lettre, je croyais la lire, je m'en récitais chaque phrase. Tout d'un coup je m'arrêtais effrayé. Je comprenais que si je devais recevoir une lettre de Gilberte, ce ne pourrait pas en tout cas être celle-là, puisque c'était moi qui venais de la composer. Et dès lors, je m'efforçais de détourner la pensée des mots que j'aurais aimé qu'elle m'écrivît, par peur, en les énonçant, d'exclure justement ceux-là, — les plus chers, les plus désirés du champ des réalisations possibles (257; Pléiade, 402).

ou encore:

[ ... ] moi qui avais d'abord aimé Gilberte, dès Combray, à cause de tout l'inconnu de sa vie, dans lequel j'aurais voulu me précipiter, m'incarner, en délaissant la mienne qui ne m'était plus rien, je pensais maintenant comme à un inestimable avantage, que de cette mienne vie trop connue, dédaignée, Gilberte pourrait devenir un jour l'humble servante, la commode et confortable collaboratrice, qui le soir, m'aidant dans mes travaux, collationnerait pour moi des brochures (258; Pléiade, 402).

L'interprétation de ces extraits en termes d'«itérativité » les rend plats et inintéressants. De toute évidence, Proust n'est pas concerné par l'aspect répétitif de ces petits événements, mais par leur importance, déjà qualifiée d'emblématique, dans la suite des jours remémorés. Cette interprétation est suggérée par Proust lui-même. C'est la seconde clef de lecture qu'il offre ainsi au lecteur. En effet, immédiatement après ce long épisode des ChampsÉlysées, et pour en faire en quelque sorte le bilan, il note: "Cette complexité du Bois de Boulogne qui en fait un lieu factice et, dans le sens zoologique ou mythologique du mot, un Jardin, je l'ai retrouvée cette année ... » (273 ; Pléiade, 414).

Un lieu factice, un Jardin mythologique. Or le temps qui lui est associé est, lui aussi, un temps mythologisé, un temps rêvé. Ni Vigneron ni Genette ne l'ont envisagé sous cet angle, préférant chercher dans ce récit des épisodes singuliers, des événements uniques, clairement délimités (exprimés au passé défini, ou passé simple) et qui peuvent être répétés, portés alors par des "imparfaits d'habitude", comme dans un récit ordinaire. Ils ont oublié, semble-t-il, qu'il s'agit de la recherche d'un temps perdu, et que ce temps perdu et recherché, que l'on essaie de faire revivre en tâtonnant, c'est le temps de la mémoire. Qu'en tout état de cause, la mémoire est présente (saint-Augustin est le premier à avoir analysé ce phénomène).

D'où une dialectique constante entre le présent — le présent à la fois du souvenir et de l'écriture - et le passé. D'où aussi la difficulté à retrouver ce passé au moyen du langage qui, par la force des choses, maintient dans le 
présent de parole. Or, pour pouvoir véritablement retrouver le passé, il faut parvenir à s'abstraire du présent et remonter aux sources du temps ou, pour utiliser une image véritablement proustienne, descendre jusqu'à ses racines. Les deux dernières pages de La Recherche ("Le temps retrouvé ») ne laissent subsister aucun doute quant à cette interprétation :

Si c'était cette notion du temps incorporé, des années passées non séparées de nous, que j'avais maintenant l'intention de mettre si fort en relief, c'est qu'à ce moment même, dans l'hôtel du prince de Guermantes, ce bruit du pas de mes parents reconduisant M. Swann, ce tintement rebondissant, ferrugineux, intarissable, criard et frais de la petite sonnette qui m'annonçait qu'enfin $\mathrm{M}$. Swann était parti et que maman allait monter, je les entendis encore, je les entendis eux-mêmes, eux situés pourtant si loin dans le passé. Alors, en pensant à tous les événements qui se plaçaient forcément entre l'instant où je les avais entendus et la matinée Guermantes, je fus effrayé de penser que c'était bien cette sonnette qui tintait encore en moi, sans que je pusse rien changer aux criaillements de son grelot, puisque ne me rappelant plus bien comment ils s'éteignaient, pour le réapprendre, pour bien l'écouter, je dus m'efforcer de ne plus entendre le son des conversations que les masques tenaient autour de moi. Pour tâcher de l'entendre de plus près, c'était en moi-même que j'étais obligé de redescendre. C'est donc que ce tintement y était toujours, et aussi, entre lui et l'instant présent tout ce passé indéfiniment déroulé que je ne savais pas que je portais. Quand elle avait tinté j'existais déjà, et depuis pour que j'entendisse encore ce tintement, il fallait qu'il n'y eût pas eu discontinuité, que je n'eusse pas un instant cessé, pris le repos de ne pas exister, de ne pas penser, de ne pas avoir conscience de moi, puisque cet instant ancien tenait encore à moi, que je pouvais encore le retrouver, retourner jusqu'à lui, rien qu'en descendant plus profondément en moi. [...]

J'éprouvais un sentiment de fatigue et d'effroi à sentir que tout ce temps si long non seulement avait, sans une interruption, été vécu, pensé, sécrété par moi, qu'il était ma vie, qu'il était moi-même, mais encore que j'avais à toute minute à le maintenir attaché à moi, qu'il me supportait, moi, juché à son sommet vertigineux, que je ne pouvais me mouvoir sans le déplacer comme je le pouvais avec lui. La date à laquelle j'entendais le bruit de la sonnette du jardin de Combray, si distant et pourtant intérieur, était un point de repère dans cette dimension énorme que je ne me savais pas avoir. J'avais le vertige de voir au-dessous de moi, en moi pourtant, comme si j'avais des lieues de hauteur, tant d'années. (Pléiade IV, 623-624).

Le passé, si l'on s'en tient aux formes de l'aspect simple du verbe, est exprimé soit au prétérit défini (passé simple), soit à l'imparfait. Le prétérit impose une vision distanciée des événements saisis dans leur perfectivité à l'horizon du temps ; c'est la raison pour laquelle c'est le temps du récit par excellence - le temps du dévidement et de la successivité des événements. Examinons de plus près le passage suivant, qui est la version originale (Grasset 1913), où dominent les imparfaits :

Tout à coup l'air se déchirait: entre le guignol et le cirque, à l'horizon embelli, sur le ciel entrouvert, je venais d'apercevoir, comme un signe fabuleux, le 
plumet bleu de Mademoiselle. Et déjà Gilberte courait à toute vitesse dans ma direction, étincelante et rouge sous un bonnet carré de fourrure, animée par le froid, le retard et le désir du jeu; un peu avant d'arriver à moi, elle se laissa glisser sur la glace et, soit pour mieux garder son équilibre, soit parce qu'elle trouvait cela plus gracieux, ou par affectation du maintien d'une patineuse, c'est les bras grands ouverts qu'elle avançait en souriant, comme si elle avait voulu m'y recevoir.

Sur 7 formes verbales personnelles, 6 sont effectivement des imparfaits ${ }^{22}$; la septième ( $\underline{\text { se laissa }}$ ) est un prétérit. J'ai déjà signalé qu'en 1919 Proust avait remplacé le premier imparfait (se déchirait) par un prétérit. Mais il aurait pu aussi remplacer par le prétérit d'autres imparfaits (notamment avançait). Moyennant quelques transformations, tout ce passage pourrait du reste être entièrement au prétérit :

Tout à coup l'air se déchira : entre le guignol et le cirque, à l'horizon embelli, sur le ciel entrouvert, $j^{\prime}$ aperçus soudain, comme un signe fabuleux, le plumet bleu de Mademoiselle. Et Gilberte se mit à courir à toute vitesse dans ma direction, étincelante et rouge sous un bonnet carré de fourrure, animée par le froid, le retard et le désir du jeu ; un peu avant d'arriver à moi, elle se laissa glisser sur la glace et, soit pour mieux garder son équilibre, soit parce qu'elle trouva cela plus gracieux, ou par affectation du maintien d'une patineuse, c'est les bras grands ouverts qu'elle avança en souriant, comme si elle eut voulu m'y recevoir.

Mais il faut convenir que, dans cette version remaniée, la tonalité proustienne a singulièrement diminué, pour ne pas dire qu'elle a complètement disparu. En recourant massivement à l'imparfait, combiné à l'emploi des marques lexicales du temps qu'on vient d'examiner, Proust cherche à abstraire les événements remémorés d'une suite chronologique trop bien marquée, afin de les revivre (et de les faire revivre) en se plongeant dans leur immanence.

On connaît l'analyse psychomécanique de l'imparfait; je n'y reviens pas $\mathrm{ici}^{23}$. Il suffira de rappeler que c'est le temps de l'imperfectif par excellence, dans la mesure où il oblige à la saisie interne de l'événement (que celui-ci soit simple ou complexe, unique ou multiple), sans que soit évoqués à la fois son commencement et sa fin. De là l'impression d'immanence, de « déjà » et de " pas encore ». En choisissant, comme il le fait, de recourir de façon marquée à l'imparfait - surtout lorsque le prétérit, dans un premier temps, viendrait plus spontanément - Proust

22. Y compris la dernière forme, celle de plus-que-parfait, considéré en psychomécanique comme l'imparfait de l'aspect dit transcendant du verbe simple, en l'occurrence vouloir. Le passé composé est le présent de ce même aspect.

23. Je renvoie à Guillaume, à Temps et verbe, bien sûr, mais aussi, et peut-être surtout à certains volumes des Leçons (par ex. les vol. 1 et 10). 
favorise cette vision de l'intérieur qu'il recherche. La présence des imparfaits correspond-elle à un "artifice", comme se plaît à le dire Vigneron, ou bien à une vision très spéciale, une vision en immanence, c'est-à-dire en intériorité, comme je le proposais plus haut ? C'est pour cette dernière hypothèse que je penche.

Dans ce long épisode des Champs-Élysées, on a vu comment le souvenir de toute une série d'après-midis d'hiver se cristallise en tel ou tel incident, qui, en tant que moment culminant, fait la synthèse de tous les autres, moins mémorables. On est dans l'immanence du temps et l'on sélectionne tel ou tel moment. Une fois de plus, il ne s'agit pas d'itération, mais de quelque chose de plus subtil. Proust lui-même, probablement sans s'en douter, offre une nouvelle clef pour la lecture, non seulement de cet épisode, mais de l'ensemble de La Recherche.

On sait qu'il a hésité sur les titres à donner aux divers volumes et à l'ensemble de l'œuvre. Les Intermittences $d u$ Cour pour désigner l'ensemble avait été envisagé. À partir de ce titre, voici ce qu'il écrit à un correspondant en mars 1913, quelques mois à peine avant la première publication de Swann : "Aimeriez-vous comme titre / Les Intermittences du Passé / Premier volume Le Temps perdu / Deuxième volume Le Temps retrouvé ?». Le choix d'«intermittences » doit retenir l'attention, car si le prétérit est, par excellence, le temps du récit suivi et de la continuité temporelle, mais en pure extériorité, l'imparfait est par excellence le temps de l'intermittence et de la discontinuité. Il y a là un paradoxe apparent qu'il convient de souligner. Une intermittence est un arrêt momentané, d'où, en médecine, l'idée d'un intervalle pendant lequel un phénomène ne se produit pas. Ce terme pourra ainsi désigner une période de répit entre deux accès de fièvre. Les "intermittences du cœur », au sens premier et médical, sont donc les absences momentanées d'une pulsion cardiaque. Proust l'emploie figurativement et il en tire «intermittences du passé ». Qu'entend-il par là ?

À mon sens, il s'agit des espaces temporels vides entre les occurrences qui occupent effectivement la mémoire, par exemple "un de ces jours de soleil » (v. citation ci-dessus) où, dans l'ensemble «jours de soleil » non présent - existant dans la mémoire latente - auquel renvoie le déictique ces par exophore mémorielle (cf. A. Joly \& Ó Kelly, Grammaire systématique de l'anglais, 1990, 438-441), un jour particulier est retenu en mémoire présente. Il s'ensuit une impression de discontinuité dans le tissu temporel, une sorte de «trou » dans la mémoire.

Les intermittences du temps (on peut généraliser à partir du passé) constituent donc en quelque sorte l'envers de l'itération (de la «fréquence») ou, si l'on préfère, l'itération en creux, le négatif de l'itération. Car l'itération est une opération positive : elle consiste à poser et à généraliser dans le temps un certain événement. Contrairement à ce que disent Vigneron et Genette, tel n'est pas le projet de Proust. 
L'itérativité, fondée sur l'acceptation du discontinu, n'est pas son affaire. Son objectif est au contraire de retrouver le temps en parvenant à reconstituer sa continuité, sa fluence originelle, ce qui exclut l'itération, qui, elle, accepte le vide. Le seul moyen de retrouver la fluence perdue est de combler les intermittences en reconstituant l'entour des événements remémorés ${ }^{24}$.

Or ce type d'opération n'est linguistiquement possible, en français, que si l'on se situe dans l'immanence des événements (simples ou complexes) grâce à la structure de la forme d'imparfait qui, on le sait, permet la focalisation sur un instant entre deux limites non actualisées, mais virtuellement présentes. Le projet, apparemment impossible, mais réalisé par Proust in extremis dans La Recherche, est d'actualiser cette présence virtuelle. De proche en proche, par petites touches successives et persévérantes, l'intégralité du temps perdu, de son commencement à sa fin — c'est-à-dire en fait l'instant de parole — pourra ainsi être restituée.

\section{Conclusion}

L'entreprise de Genette, pour intéressante qu'elle soit dans d'autres domaines - en particulier celui de l'analyse des macro-structures narratives - me paraît donc, dès le départ, vouée à l'échec. En effet, elle ne part pas du texte, mais d'une idée de ce qu'est ou de que devrait être un texte. Elle est fondée sur des concepts empruntés et importés sans avoir été préalablement expérimentés, vérifiés, ajustés. L'emprunt analogique à l'analyse linguistique du verbe illustre parfaitement cette démarche exclusivement hypothético-déductive. Rien d'étonnant à ce que les grilles d'analyse qu'elle produit ne parviennent à rendre compte ni de la microstructure des textes, ni surtout de leur écriture. Comment prétendre pouvoir analyser Proust d'une façon quelque peu crédible si l'on ne s'est pas préalablement donné le temps de réfléchir sur la nature des matériaux linguistiques utilisés, par exemple à propos du verbe dans le passage qui vient d'être examiné, sur l'aspect, la voix, le mode et les temps? L'analyse textuelle commence par l'analyse linguistique — parfois une analyse strictement "grammaticale »-, c'est là un truisme qu'il n'est jamais inutile de rappeler. Selon Gustave Guillaume, en effet, « il faut toujours en revenir au dire, objectif constant du langage ».

Équipe ERIS, Babel, EA 2649

\section{Bliographie complémentaire}

Adam, Jean-Michel (1992), Les textes : types et prototypes (récit, description, argumentation, explication et dialogue), Nathan, Paris.

Alain (1945), En lisant Dickens, Gallimard, Paris.

24. Comme, en termes de dentisterie, on « reconstitue » une dent perdue autour de ce qu'il en reste. 
Auerbach, Erich ([1946], trad. 1968), Mimésis, nrf, Gallimard, Paris.

Borges, Jorge Luis ([1956], trad. 1957), Fictions, Gallimard, Paris.

Bourneuf, Roland \& Ouellet, Réal ([1972] 19956), L'univers du roman, puf, Paris.

Bremond, Claude (1973), Logique du récit, Seuil, Paris.

Butor, Michel (1960/1964), Essais sur le roman, nrf, Gallimard.

Chion, Michel (1985), Écrire un scénario, Cahiers du Cinéma, I.N.A., Paris.

Dumézil, Georges (1970), Du mythe au roman, Presses Universitaires de France, Paris.

Genette, Gérard (1966-2002), Figures, essais (Figures I à Figures V), Seuil, Paris.

Genette, G. ([1979] 2004), Introduction à l'architexte, Seuil, coll. «Points essais », Paris.

Genette, G. (1983), Nouveau Discours du récit, Seuil, coll. « Poétique », Paris.

Genette, G. (2007), Discours du récit, Seuil, coll « Points essais », Paris, 2007. (Rassemble Discours du récit (Figures III, p. 71-273) et Nouveau Discours du récit).

Girard, René (1961), Mensonge romantique et vérité romanesque, Grasset, Paris.

James, Henry (trad. 1954), Carnets (Notebooks), Éditions Denoël, Paris.

Jauss, Hans Robert ([1972], trad. 1978), Pour une esthétique de la réception, nrf, Gallimard, Paris.

Jolles, André ([1930] 1972), Formes simples, Seuil, Paris.

Joly, André \& Ó Kelly, Dairine (1990), Grammaire systématique de l'anglais, Nathan, Paris.

Lejeune, Philippe (1975), Le pacte autobiographique, Éditions du Seuil, Paris.

Magny, Claude-Edmonde (1950), Histoire du roman français depuis 1918, tome 1, Seuil, Paris.

Merleau-Ponty, Maurice (1969), La prose du monde, nrf, Gallimard, Paris.

Mitterand, Henri (1980), Le discours du roman, puf, Paris.

Mitterand, H. (1998), Le roman à l'œuvre. Genèse et valeurs, puf, Paris.

Ó Kelly, Dairine (1990), v. Joly 1990.

(1995), «Temporalité et textualité : le cas Weinrich», Modèles linguistiques, tome XVI, vol. 32, 145,165, Éditions des Dauphins, Toulon.

Ouvrage collectif (1965), Que peut la littérature? L'inédit 10/18, Paris.

Weinrich, Harald (1964), Le temps, Seuil, Paris.

Zumthor, Paul (1975), Langue, texte, énigme, Seuil, Paris. 